Reprod. Nutr. Dévelop., 1986, 26 (1 B), 329-330.

\title{
Influence d'un faible niveau d'ingestion sur la digestion et le comportement alimentaire chez la vache recevant un régime très digestible
}

M. DOREAU, A. I. LOMRI, K. ADINGRA

Laboratoire de la Lactation,

I.N.R.A. Theix, 63122 Ceyrat, France.

Summary. Decreasing the level of feeding a maize silage-concentrate diet sharply improved organic matter digestibility (1 unit $/ \mathrm{kg} \mathrm{DM}$ intake). The rumen retention time of the digesta and chewing efficiency were increased, but microbial activity was not modified.

L'influence de faibles niveaux d'ingestion sur la digestion des rations du ruminant a été essentiellement étudiée pour des régimes à base de fourrages peu digestibles. Nous l'avons analysée chez la vache laitière avec un régime de valeur nutritive élevée.

Matériel et méthodes. Trois vaches en fin de lactation, fistulées du rumen et du duodénum, ont reçu, selon un carré latin, $12 \mathrm{~kg}$ (quantité légèrement inférieure au niveau de consommation volontaire), 8 ou $4 \mathrm{~kg}$ de matière sèche (MS) d'une ration comprenant $70 \%$ d'ensilage de maïs et $30 \%$ de concentré, bien pourvue en azote fermentescible, et dont les teneurs en matières azotées et cellulose brute étaient de 15,5 et $15,8 \%$ de la MS. Une des vaches ayant été malade lors d'une période ( $8 \mathrm{~kg}$ de MS), les données correspondantes ont été estimées et les résultats soumis à une analyse de variance à deux voies : traitement et animal.

Les mesures suivantes ont été effectuées : digestibilité par collecte des fèces, cinétique de disparition in sacco de tiges de maïs ensilées broyées ; $\mathrm{pH}$, concentration et composition des acides gras volatils (AGV) du rumen, temps de séjour dans le rumen et la totalité du tractus digestif par analyse de l'excrétion fécale de particules colorées, quantité de contenu frais et sec du rumen par vidage, taille moyenne des particules quittant les pré-estomacs par granulométrie du contenu duodénal. Les activités alimentaires et méryciques ont été mesurées par enregistrement des mouvements de la mâchoire ; la durée de rumination a été calculée en tenant compte du taux de rumination effective, proportion de mastication durant une période de rumination, obtenue par agrandissement photographique.

Résultats et discussion. L'accroissement de la digestibilité de la matière organique (tabl. 1) a été plus important pour une même diminution du niveau d'ingestion que ce qui avait été mesuré avec ce type de régime par Tyrrell et Moe (1972), mais permet d'expliquer la digestibilité élevée observée en fin de gestation par Doreau et Rémond (1982). L'effet du niveau alimentaire sur la digestibilité ne semble pas dû à une modification de l'activité microbienne : les pourcentages de MS disparue des sachets ont été indépendants des quantités ingérées, et égaux en moyenne à $43,3-50,6-61,7-72,7$ et $78,1 \%$ après $6,12,24,48$ et $72 \mathrm{~h}$ de séjour dans le rumen. De plus, le $\mathrm{pH}$ et les proportions relatives d'acétate, de propionate 
et de butyrate n'ont pas été modifiés mais la concentration des AGV a évolué dans le même sens que les quantités ingérées. La diminution du niveau d'ingestion n'a pas modifié le transit intestinal mais a accru fortement le temps de séjour des digesta dans le rumen, ce qui peut expliquer les variations de digestibilité, puisque la cinétique in sacco a mis en évidence que la digestion ruminale se poursuit au moins pendant $72 \mathrm{~h}$. Le temps de séjour dans le rumen a augmenté plus entre 8 et $4 \mathrm{~kg}$ de MS qu'entre 12 et $8 \mathrm{~kg}$ de MS, ce qui a correspondu à une moindre réduction du contenu sec du rumen. A $4 \mathrm{~kg}$ de $\mathrm{MS}$, la teneur en eau du contenu de rumen s'est accrue ce qui a eu pour conséquence de ne pas réduire excessivement le volume occupé par les digesta. L'accélération du transit avec l'accroissement du niveau d'ingestion n'a pas entraîné d'augmentation de la taille moyenne des particules entrant dans le duodénum; celle-ci semble beaucoup plus liée au temps passé à mastiquer un $\mathrm{kg}$ de MS. Les durées d'ingestion et de rumination ont été beaucoup plus réduites entre 12 et $8 \mathrm{~kg}$ de MS qu'entre 8 et $4 \mathrm{~kg}$ de MS. A ce dernier niveau, la rumination a été perturbée pour deux des trois vaches. Leurs taux de rumination effective ont été de 67 et $70 \%$, alors que la moyenne des autres taux a été de $92 \%$. Une telle perturbation se rapproche de la « rumination de transition » décrite par Welch et Smith (1968) en cas de jeûne.

TABL. 1. - Effet du niveau d'ingestion sur la digestion et la mastication.

\begin{tabular}{|c|c|c|c|c|}
\hline Niveau d'ingestion ( $\mathrm{kg}$ de $\mathrm{MS} / \mathrm{j}$ ) & 12 & 8 & 4 & $\begin{array}{l}\text { Effet } \\
\text { statistique }\end{array}$ \\
\hline Digestibilité de la matière organique $(\%)$ & 73,8 & 77,4 & 80,9 & ** \\
\hline $\begin{array}{l}\text { Concentration en AGV avant repas }(\mathrm{mM} / \mathrm{l}) \\
\text { Temps de séjour moyen des grosses particules }(\mathrm{h}) \text { : }\end{array}$ & 64,1 & 50,7 & 46,8 & - \\
\hline - dans le tractus digestif & 63 & 77 & 99 & ** \\
\hline - dans le rumen & 30 & 43 & 63 & $*$ * \\
\hline Contenu frais de rumen $(\mathrm{kg})$ & 71,6 & 46,3 & 46,8 & $*$ \\
\hline Contenu sec de rumen $(\mathrm{kg})$ & 7,43 & 4,65 & 3,47 & * \\
\hline $\begin{array}{l}\text { Taille moyenne des particules au niveau duodénal } \\
\text { (mm) }\end{array}$ & 0,23 & 0,40 & 0,34 & - \\
\hline Durée d'ingestion $(\mathrm{min} / \mathrm{j})$ & 209 & 73 & 45 & ** \\
\hline Durée effective de rumination $(\mathrm{min} / \mathrm{j})$ & 331 & 156 & 134 & * \\
\hline Durée unitaire de mastication ( $\mathrm{min} / \mathrm{kg} \mathrm{MS}$ ) & 45 & 29 & 44 & - \\
\hline
\end{tabular}

Seuils de signification des effets statistiques : ${ }^{*} 5 \%,{ }^{* *} 1 \%$.

En conclusion, l'effet du niveau d'ingestion sur la digestibilité d'un régime à base d'ensilage de maïs est aussi important qu'avec des fourrages plus pauvres, même à des niveaux d'ingestion très bas, essentiellement en raison des variations du temps de séjour dans le rumen.

Doreau M., Rémond B., 1982. Comportement alimentaire et utilisation digestive d'une ration de composition constante chez la vache laitière en fin de gestation et en début de lactation. Reprod. Nutr. Dévelop., 22, 307-324.

Tyrrell H. F., Moe P. W., 1972. Net energy value for lactation of a high and low concentrate ration containing corn silage. J. Dairy Sci., 55, 1106-1112.

Welch J. G., Smith A. M., 1968. Influence of fasting on rumination activity in sheep. J. anim. sci., 27. 1734-1737. 